\title{
Glassy solids come of age
}

\section{There is a promise of some progress in the understanding of the differences between glasses on the one hand and other kinds of solids, or liquids, on the other.}

Is the glassy solid about to come of age? This is the possibility suggested by the apparently boundless interest, just now, in disordered conditions of the solid state, the manifestations of which apparently multiply as the weeks go by. Thus the possibility that quasicrystals, the structures of solids with apparently five-fold symmetry, are glassy structures remains (see M. Widom's News and Views article on page 19 of this issue).

So does the possibility that the new ceramic superconductors, with their nonstoichiometric chemical composition, may be solid solutions of one phase in another, with clumps of one phase extending over very small numbers of inter-atomic distances. There has also been some gentle excitement about the amorphous forms of ice formed at high pressure (see last week's Nature 326, 823; 1987). In the circumstances, it is more than ever a pity that so little has been done to put the theory of glasses on a footing that is at once firm and accessible.

There is no obvious room for complaint. Nobody will claim that the problem of understanding what a glass is like can be simple. Indeed, the definition we were all given at school, that a glass is a liquid capable of supporting a shear stress, is still about the best there is; the obvious defect is that this phenomological statement by itself provides no pointers to the atomic structure of the glassy state.

The other conspicuous part of our schoolroom lore, that glasses are inherently unstable relative to some more ordered solid state, and remain metastable only for kinetic reasons - the transition to crystallinity is exceedingly slow - cannot apply to all glasses, polymer glasses, for example, where separate molecules may be inextricably tangled with each other, so that the solids are irretrievably disordered.

But there is one practical sense in which the formation of glasses is a kinetic phenomenon: to turn a supercooled liquid into a glass, the rate of cooling has to be great enough to ensure that some degree of departure from equilibrium will be incorporated into the solid.

None of this implies that people have been indifferent to the need for understanding. That there is something special about the glassy state, and therefore much in need of definition and description, has been clear for many years, not merely because people have been asking how it can be that materials that can support shear stress nevertheless creep, if comparatively readily (which is why windowpanes in old houses are thicker at the bottom than the top), but because of empirical observations at low temperatures which, among other things, show that the specific heat is a linear rather than a Debye-type cubic function. (The moral is that there is more scope for sucking up low-energy quanta in a glass than in a classically vibrating lattice.)

The wind now seems to be blowing in a direction much favoured for much more than ten years - towards that picture of the microscopic structure of a glass in which small well-ordered groups of atoms are oriented relative to each other in a random fashion and, therefore, connected together by equally random bonds. This arrangement has the virtue of being in agreement with $\mathrm{X}$-ray diffraction measurements to the extent that there is shortrange order of the kind found in powder diffraction photographs, but no more long-range order than can be found in a liquid. (Resistance to shear-stress arises from the mere existence of linking bonds.) The obvious snags are that randomly arranged bonds may imply severe local energetic penalties and that there are geometrical problems as well; often the natural small-scale units will be tetrahedral units, but there is no way of filling space with structures like that, any more than there is a way of tiling the twodimensional plane with pentagons.

The much more serious difficulty is that there is no obvious way of calculating the properties of a glass built thus from small ordered groups of atoms. What conviction can a theory carry if it cannot be used to make some kind of prediction, even of the properties of some well-studied glass? That, for ten years, has been the hang-up. Now, with a little luck, there may be a way out of the fix.

R. Kree of the University of Düsseldorf, L.A.Turski of the Polish Academy of Sciences and A. Zippelius of the solidstate institute at the West German nuclear research centre at Jülich in Nordrheinwesfalia have just published (Phys. Rev. Lett. 58, 1656; 1987) a seductive account of how such a problem might be handled. If a glass is a system in which well-ordered groups of atoms which are randomly oriented interact with identical neighbouring groups of atoms by means of randomly drawn bonds, why not rep- resent the entire system by that elaboration of the Ising lattice (a three-dimensional lattice with either parallel or antiparallel magnets on every vertex) called the Potts model, in which the interacting elements at the vertices may have a denumerably infinite number of orientations?

To be sure, the simple problem of the general Ising lattice has not yet been solved, but there are tricks (computational, but also topological) that can finesse that difficulty.

Kree and his associates give an impressive prospectus of their achievement. What Ising lattice people attempt is usually the calculation of the thermodynamic partition function, from which the usual thermodynamic quantities can be derived, from a statement of the geometry of the problem. Kree et al. build into their analysis a consideration of the interaction between the local orientation of a group of atoms in a glass (from which it may be possible to calculate the shear strength of glasses and its variation with temperature), which has always been a technical option from which most of the practitioners have shrunk.

Tantalizingly, the details of the calculations are not yet available, but only the general conclusions claimed for them. Briefly, the narrative continues, there is such a thing as the transition to a glassy state, the transition temperature is a function of the degree of disorder frozen into the solid but there is no latent heat at the glass transition, whether or not there are other discontinuities, nor is there a change of specific volume.

All of us will live more happily with these conclusions when the intricacies of the argument have been written up. No doubt, that will not be long from now. Meanwhile, those working in the field will be reflecting that, while the Potts model as such is so hard to handle that it offers very little in the way of rapid progress, the Ising lattice has yet again been shown to be a durable and adaptable model of a huge variety of physical systems. Now the obvious need is to find a physical system, a real glass, that will conform with what Kree et al. promise. The plain truth is that Kree et al. have suggested powerfully a way in which a familiar model of the crystalline state may be adapted to an understanding of puzzling disorder. Watch this space, as they say.

John Maddox 